\title{
Model Reduction Design of Multivariable Aircraft System Using Fast Haar Transform Algorithm
}

\author{
Joon-Hoon Park and Ryum-Duk Oh* \\ Department of Control and Instrumentation Engineering, Korea National \\ University of Transportation, Chungju-si, Chungbuk-do, (380-702), South Korea \\ *Department of Software Engineering, Korea National University of \\ Transportation, Chungju-si, Chungbuk-do, (380-702), South Korea
}

\begin{abstract}
In this paper, a model reduction method for high-order multivariable aircraft system is presented based on the fast Haar transform algorithm. The transfer function is one of the important factors that determine the characteristics of a system. This transfer function of the MIMO (Multiple Inputs and Multiple Outputs) system is easily derived from the SISO system. However, a transfer function of the MIMO system often contains high order and has little effect on the system response. Thus, it is desirable to find a reduced model from a given high-order MIMO system. Therefore the analysis and design effort can be reduced. The Haar function set forms a complete set of orthogonal rectangular functions similar in several respects to the Walsh functions. If factorization algorithm is employed to the conventional Haar transform then the fast Haar transform can be obtained. The model reduction method adopted in this paper is that of system approximation algorithm using the fast Haar transform. This approach provides more convenient and efficient results.
\end{abstract}

Keywords: model reduction, multiple inputs and outputs system, high-order system, fast Haar transform algorithm, operational matrix

\section{Introduction}

Related to the transfer function, it can be obtained by the Laplace transform of the differential equations and the characteristic equation of system are defined by setting the denominator polynomial of the transfer function to zero. The addition of poles and zeros or cancellation of undesirable poles and zeros of the transfer function are often necessary to achieve satisfactory analysis and design of the control system. It is important to determine the poles that have an important effect on the system response. However, highorder transfer function system open has insignificant poles that have little effect on the system performance and response. In this case, less significant poles can be disregarded and the system can be approximated as a low-order system. Therefore, in this reduced model by neglecting the less important poles case, the time domain response that is based on the characteristic equation of control system shows dynamic properties similar to those of the original high-order system. Therefore, simplification and reduction of high-order system are among the major concerns in the system analysis and design. In the present paper, fast Haar functions and transform that were described by Alfred Haar are used for model reduction design of the aircraft MIMO system. The fast transform has the advantage of faster processing speed than the general transform method. The algorithm and method proposed in this paper are useful for system approximation and reduction and

Received (June 22, 2017), Review Result (October 15, 2017), Accepted (October 22, 2017)

${ }^{*}$ Corresponding Author 
the results are accurate and convenient.

\section{Haar Functions}

The Haar functions form an orthogonal and orthonormal system of periodic square waves. The amplitude value of these square waves does not have uniform values, but assume a limited set of values, $0, \pm 1, \pm \sqrt{2}$ etc [1]. If we consider the time base to be defined as $0 \leq \mathrm{t} \leq 1$ then, the set of Haar functions can be described as follows:

$$
\begin{gathered}
\operatorname{Har}(0, t)=1 \quad \text { for } 0 \leq t \leq 1 \\
\operatorname{Har}(1, t)=\left\{\begin{array}{cc}
1 & \text { for } 0 \leq t \leq \frac{1}{2} \\
-1 & \text { for } \frac{1}{2} \leq t \leq 1
\end{array}\right. \\
\operatorname{Har}(2, t)=\left\{\begin{array}{rrr}
\sqrt{2} & \text { for } 0 \leq t \leq \frac{1}{4} \\
-\sqrt{2} & \text { for } \frac{1}{4} \leq t \leq \frac{1}{2} \\
0 & \text { for } & \frac{1}{2} \leq t \leq 1
\end{array} \quad\right. \\
\operatorname{Har}(i, t)=\left\{\begin{array}{rrr}
\sqrt{2^{p}} & \text { for } & \frac{n}{2^{p}} \leq t \leq \frac{n+\frac{1}{2}}{2^{p}} \\
0 & -\sqrt{2^{p}} & \text { for } \quad\left(n+\frac{1}{2}\right) / 2^{p} \leq t \leq(n+1) / 2^{p} \\
0 & \text { elsewhere }
\end{array}\right.
\end{gathered}
$$

where $i=2^{p}+n, p=0,1,2, \ldots, \log _{2} \frac{m}{2}$ and $n=0,1, \ldots, 2^{p}-1$. In Figure 1 , the first eight Haar functions are shown.

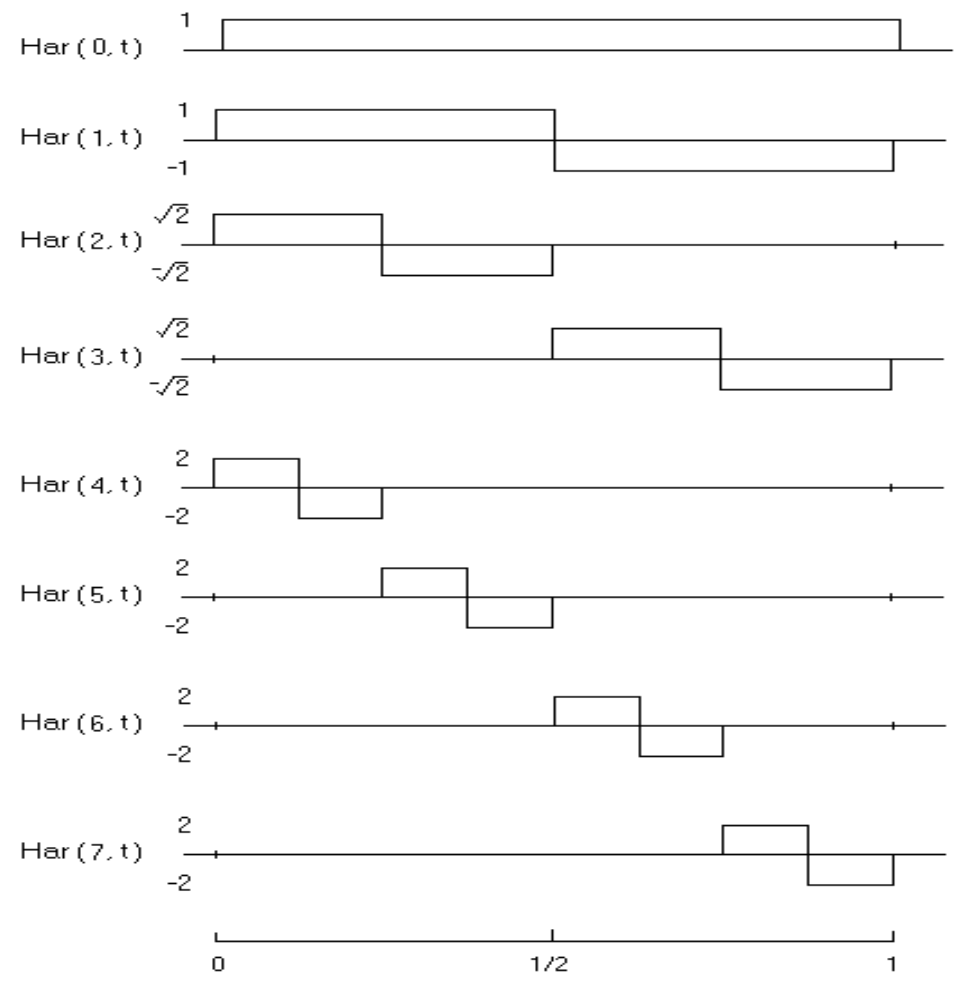

Figure 1. The First Eight Haar Functions 


\section{General Haar Operational Matrix and Transform}

The integration of the Haar functions with respect to time $t$ form a ramp and triangular waveforms. And it is related approximately to the Haar functions operational matrix itself. We can describe the Haar operational matrix $P$ in a mathematical form [2][3]. The subscript in Eq. (4) means dimension of square matrix $P$. In this case for example the $P_{(4 x 4)}$ is shown in Eq. (5).

$$
\begin{gathered}
\int_{0}^{t} H(i, \tau) d \tau \cong P H(i, t) \\
P_{(m \times m)}=\left[\begin{array}{ccr}
P_{\left(\frac{m}{2} \times \frac{m}{2}\right)} & -\frac{1}{\sqrt{2}} m^{\frac{-3}{2}} H_{\left(\frac{m}{2} \times \frac{m}{2}\right)} \\
\frac{1}{\sqrt{2}} m^{\frac{-3}{2}} H^{T}\left(\frac{m}{2} \times \frac{m}{2}\right) & 0 & \left(\frac{m}{2} \times \frac{m}{2}\right)
\end{array}\right] \\
P_{(4 \times 4)}=\left[\begin{array}{cccr}
1 / 2 & -1 / 4 & -\sqrt{2} / 16 & -\sqrt{2} / 16 \\
1 / 4 & 0 & \sqrt{2} / 16 & -\sqrt{2} / 16 \\
\sqrt{2} / 16 & -\sqrt{2} / 16 & 0 & 0 \\
\sqrt{2} / 16 & \sqrt{2} / 16 & 0 & 0
\end{array}\right]
\end{gathered}
$$

A function $f(t)$ is absolutely integral in $t \in[0,1)$, then it can be expanded in an infinite series using Haar transform.

$$
f(t)=f_{0} H_{0}(t)+f_{1} H_{1}(t)+\cdots=\sum_{i=0}^{\infty} f_{i} H_{i}(t)
$$

where $f_{i}$ is the $i$ th sequentially ordered coefficient of the Haar functions expansion of function $f(t)$ and $H_{i}(t)$ is the $i$ th ordered Haar functions. The coefficient of the Haar functions expansion is given in Eq. (7). To obtain the coefficients of Haar transform, the mean square error $\varepsilon$ must be minimized [4].

$$
\begin{aligned}
& f_{i}=\int_{0}^{1} f(t) H(i, t) d t \\
& \varepsilon=\int_{0}^{1}\left[f(t)-\sum_{i=0}^{m-1} f_{i} H(i, t)\right]^{2} d t
\end{aligned}
$$

If $f(t)=t^{2}$, then $f(t)$ can be expressed using Haar operational matrix $P$, we can get approximated $f(t)$ from Eq. (9) and Figure 2.

$$
\begin{gathered}
f(t)=2 \int_{0}^{t} f_{i} H_{i}(\tau) d \tau \\
\quad=0.3359 H_{0}(t)-0.25 H_{1}(t)-0.0442 H_{2}(t)-0.1326 H_{3}(t) \\
-0.0078 H_{4}(t)-0.023 H_{5}(t)-0.039 H_{6}(t)-0.0547 H_{7}(t)
\end{gathered}
$$




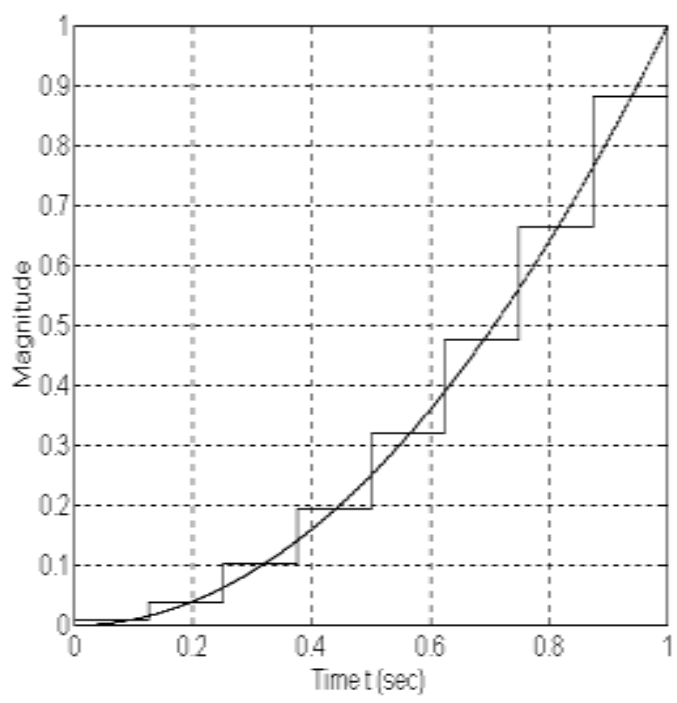

Figure 2. Haar Transform of $f(t)=t^{2}$ with Operational Matrix $P$

\section{Fast Haar Transform Algorithm}

Due to its computational simplicity, the fast Haar transform algorithm yields the fastest linear transform. This transform method is particularly valuable for a large system to have multiple inputs and outputs [5]. The computational signal flow graph for 8 points fast Haar transform is shown in Figure 3. Since the operational matrix of fast Haar transform is not symmetrical, the inverse fast Haar transform is required Figure 4. In Figure 4, the solid lines show addition and the dotted lines show subtraction at each point.

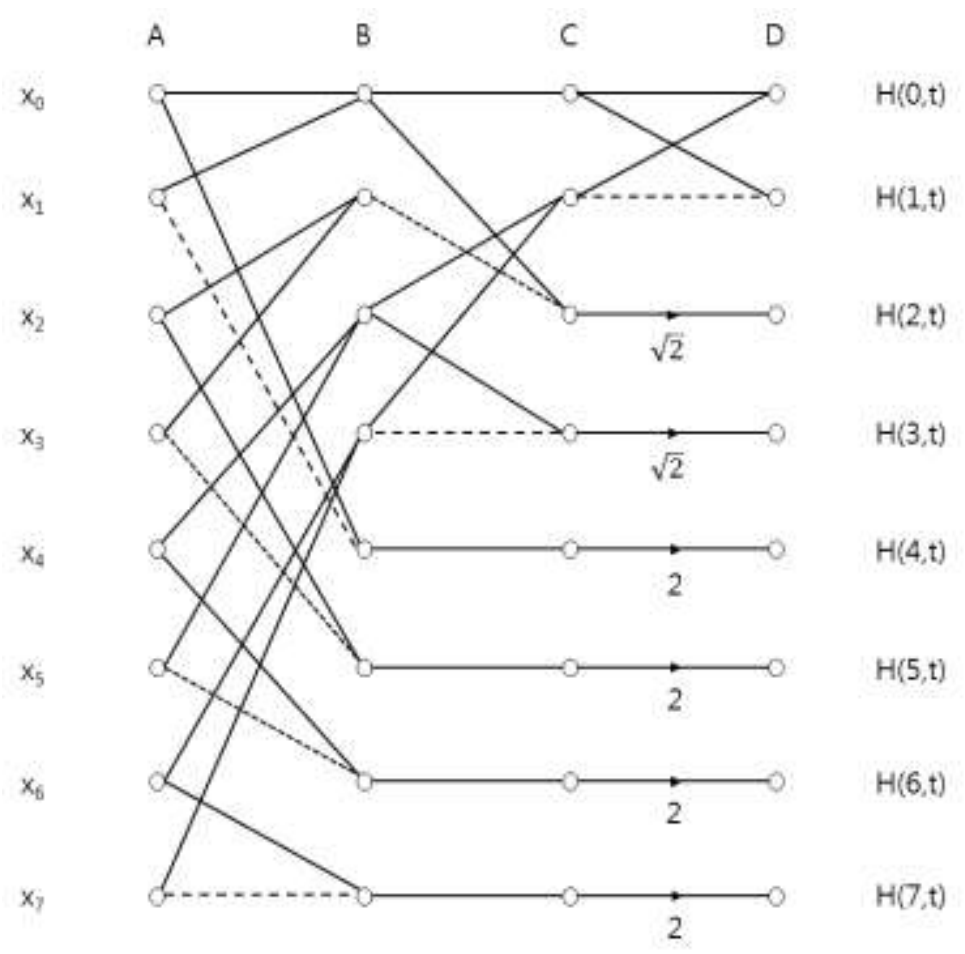

Figure 3. Computational Signal Flow Graph of the Fast Haar Transform with $n=8$ 


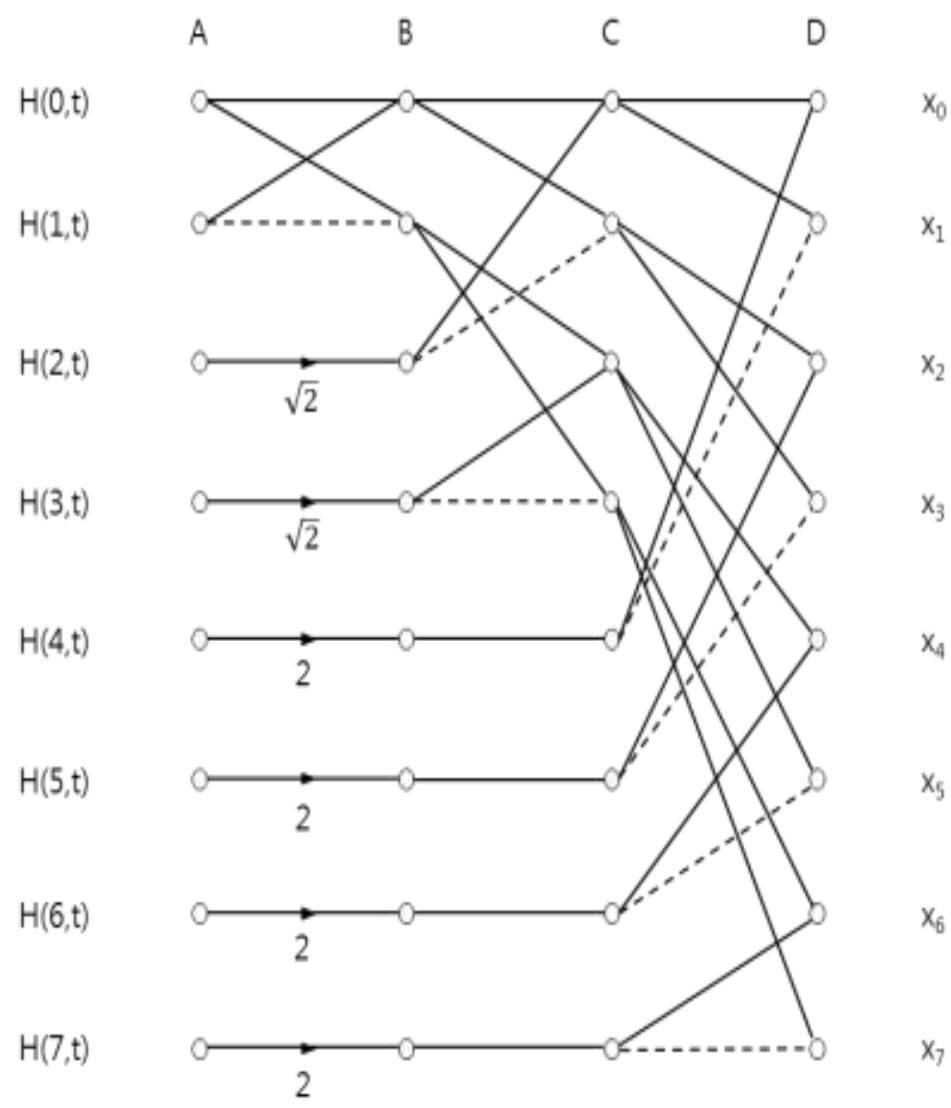

\section{Figure 4. Computational Signal Flow Graph of the Inverse Fast Haar Transform with $n=8$}

Multiplication of the sum or differences by 1 or $\sqrt{2}$ is indicated in the graph. It can be seen that, in each step of the calculation, half the points require no further calculation [6]. The total number of addition and subtraction can be stated as Eq. (10). The transform time is depends on the number of terms $\mathrm{N}$. The fast Haar transform has less complicated computational lines as compared to the general Haar transform. Therefore, the fast Haar transform is better than the general Haar transform in terms of computational time and efficiency.

$$
N+\frac{N}{2}+\frac{N}{4}+\cdots+2=2(N-1)
$$

Although the fast Haar transform algorithm has the fastest computation advantage, it may not be the most desirable, because the transform process is accomplished by means of a structured system. This system hardware for transform and calculation depends on processor hardware and computational logic. Many real time applications, particularly those concerned with structured system or processor hardware require special purpose hardware either to accomplish the required operating speed or to accomplish system efficiency. Hybrid transform which combines the advantages of the Haar and Walsh transform has been developed by Rao as shown in Figure 5 [7]. 


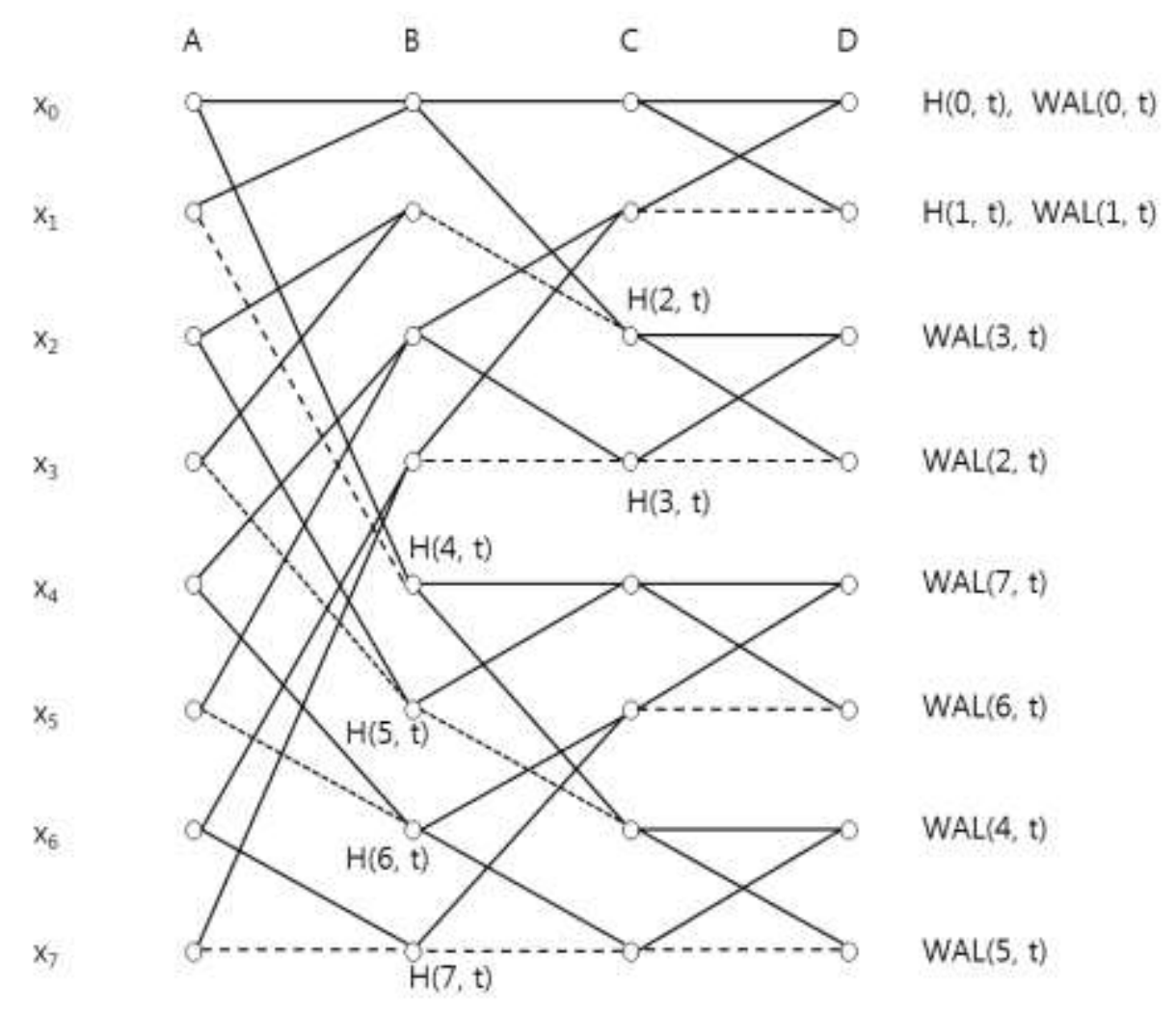

Figure 5. Computational Signal Flow Graph of the Haar and Walsh Transform

\section{Model Reduction Design of High-Order MIMO System}

In general, a linear time invariant control system can be modeled mathematically using the transfer function derived from a differential equation. The transfer function is used for the analysis of the control system. For examples, even though controllability and observability are concepts and tools of the modern control theory, they are closely related to classical properties of the transfer function. The transfer function is also widely used in electric network systems, mechanical systems and electrical systems. This transfer function $G(s)$ is related to the Laplace transform of the system input and output. The definition of the transfer function is easily extended to a system with multiple inputs and outputs that is called MIMO or a multivariable system [8]. In a MIMO system, a differential equation of the form of Eq. (11) may be used to describe the relationship between a pair of input and output variables [9].

$$
\begin{aligned}
& \frac{d^{n} y(t)}{d t^{n}}+a_{n-1} \frac{d^{n-1} y(t)}{d t^{n-1}}+\cdots+a_{1} \frac{d y(t)}{d t}+a_{0} y(t) \\
& =b_{m} \frac{d^{m} y(t)}{d t^{m}}+b_{m-1} \frac{d^{m-1} y(t)}{d t^{m-1}}+\cdots+b_{1} \frac{d y(t)}{d t}+b_{0} y(t)
\end{aligned}
$$

Figure 6 shows, the block diagram representation of a multivariable system with $p$ inputs and $q$ outputs. 


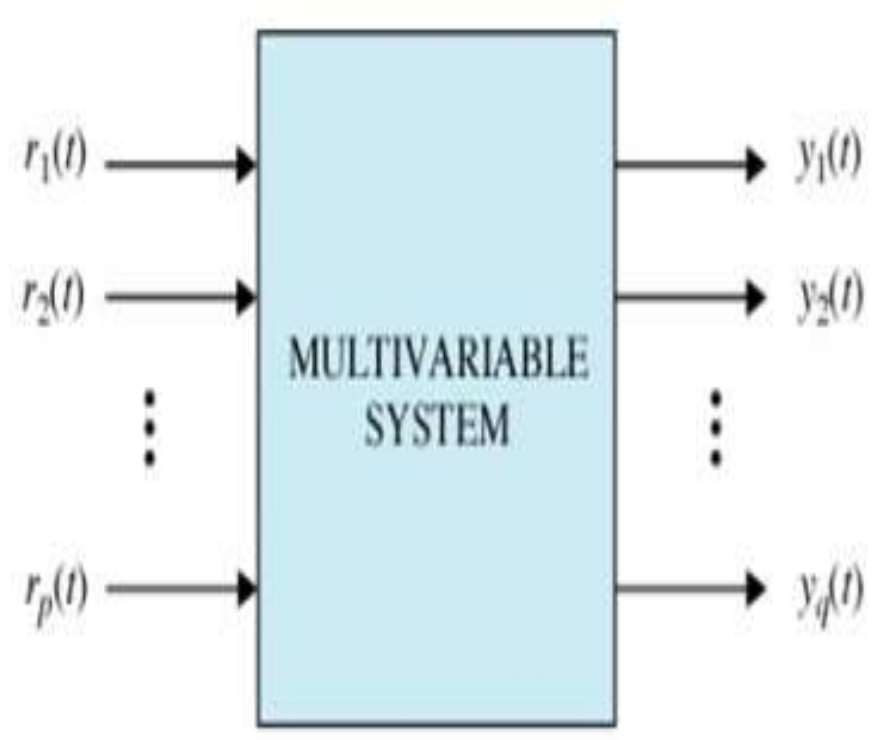

\section{Figure 6. Block Diagram Representation of a Multivariable System}

Normally, this MIMO system transfer function with $p$ inputs and $q$ outputs is expressed in matrix form $G_{M}(s)$.

$$
G_{M}(s)=\left[\begin{array}{cccc}
G_{11}(s) & G_{12}(s) & \cdots & G_{1 p}(s) \\
G_{21}(s) & G_{22}(s) & \cdots & G_{2 p}(s) \\
\vdots & \vdots & \cdots & \vdots \\
G_{q 1}(s) & G_{q 2}(s) & \cdots & G_{q p}(s)
\end{array}\right]
$$

The model reduction design is based on the theory that the frequency responses of high and low order system must be similar. Let the high order transfer function be written by Eq. (13) and the transfer function of the approximating low order be represented by Eq. (14).

$$
\begin{aligned}
G_{i j}(\mathrm{~s}) & =k \frac{1+a_{1} s+a_{2} s^{2}+\cdots+a_{m} s^{m}}{1+b_{1} s+b_{2} s^{2}+\cdots+a_{n} s^{n}} \\
\widehat{G}_{i j}(\mathrm{~s}) & =k \frac{1+c_{1} s+c_{2} s^{2}+\cdots+c_{q} s^{q}}{1+d_{1}+d_{2} s^{2}+\cdots+d_{p} s^{p}}
\end{aligned}
$$

where $n \geq m, n \geq p \geq q$. When $s=j w$ is applied to above equations, thus we can obtain Eq. (15) and (16) respectively.

$$
\begin{gathered}
G_{i j}(j w)=k \frac{\left(1-a_{2} \omega^{2}+a_{4} \omega^{4}-\cdots\right)}{\left(1-b_{2} \omega^{2}+b_{4} \omega^{4}-\cdots\right)}+\frac{j w\left(a_{1}-a_{3} \omega^{2}+a_{5} \omega^{4}-\cdots\right)}{j w\left(b_{1}-b_{3} \omega^{2}+b_{5} \omega^{4}-\cdots\right)}=k \frac{\alpha(w)+j w \beta(w)}{\gamma(w)+j w \delta(w)} \\
\widehat{G}_{i j}(j w)=k \frac{\left(1-c_{2} \omega^{2}+c \omega^{4}-\cdots\right)}{\left(1-d_{2} \omega^{2}+d_{4} \omega^{4}-\cdots\right)}+\frac{j w\left(c_{1}-c_{3} \omega^{2}+c_{5} \omega^{4}-\cdots\right)}{j w\left(d_{1}-d_{3} \omega^{2}+d_{5} \omega^{4}-\cdots\right)}=k \frac{\widehat{\alpha}(w)+j w \widehat{\beta}(w)}{\widehat{\gamma}(w)+j w \widehat{\delta}(w)}
\end{gathered}
$$

The zero frequency gain $k$ of the two transfer functions is the same. Thus, we can obtain the similar relationship of Eq. (17) to determine the unknown low order $\hat{G}_{i j}(s)$ from the original high order $G_{i j}(s)$ [10].

$$
\left|G_{i j}(j w)\right|^{2}=\left|\hat{G}_{i j}(j w)\right|^{2}=1 \quad \text { for } 0 \leq \omega \leq \infty
$$


This similarity condition means that the amplitude characteristics of the two systems in the frequency domain and the time responses of the two systems are similar. Then, we can obtain approximating solutions for the low-order transfer function by meeting the similarity condition. We can apply the fast Haar transform to the high and low order MIMO system transfer function $G_{i j}(s)$ and $\hat{G}_{i j}(s)$. We can also approximate high- order transfer function as low-order transfer function $\hat{G}_{i j}(s)$ based on the similarity condition and the fast Haar transforming algorithm [11]. Now we can express Eq. (16) as follows and apply the fast Haar transform to determine the coefficients.

$$
\begin{gathered}
\hat{\alpha}(w)=\sum_{i=0}^{n-1} \hat{\alpha}_{i} F H_{i}(w), \hat{\alpha}_{i}=\int_{0}^{1} \alpha(w) F H_{i}(t) d t \\
\hat{\beta}(\mathrm{w})=\sum_{i=0}^{n-1} \hat{\beta}_{i} F H_{i}(w), \hat{\beta}_{i}=\int_{0}^{1} \beta(w) F H_{i}(t) d t \\
\hat{\gamma}(\mathrm{w})=\sum_{i=0}^{n-1} \hat{\gamma}_{i} F H_{i}(w), \quad \hat{\gamma}_{i}=\int_{0}^{1} \gamma(w) F H_{i}(t) d t \\
\hat{\delta}(\mathrm{w})=\sum_{i=0}^{n-1} \hat{\delta}_{i} F H_{i}(w), \hat{\delta}_{i}=\int_{0}^{1} \delta(w) F H_{i}(t) d t
\end{gathered}
$$

Therefore, we can define the coefficients of low-order transfer function $\hat{G}_{i j}(s)$ from the original high-order MIMO system using the fast Haar transform. This method is useful and convenient for simplification design of the high-order MIMO system.

\section{Simulation}

Examples of MIMO systems can be found in a variety fields and plentiful practice. For example, in an automobile engine system there are two inputs of steering and accelerator, and two outputs of heading and speed. These two controls and two outputs are mutually independent. Another example is an aircraft turbo propeller engine shown in Figure 7 [9]. In this case, inputs are the fuel rate and the propeller blade angle and outputs are the speed of rotation of engine and the turbine inlet temperature.

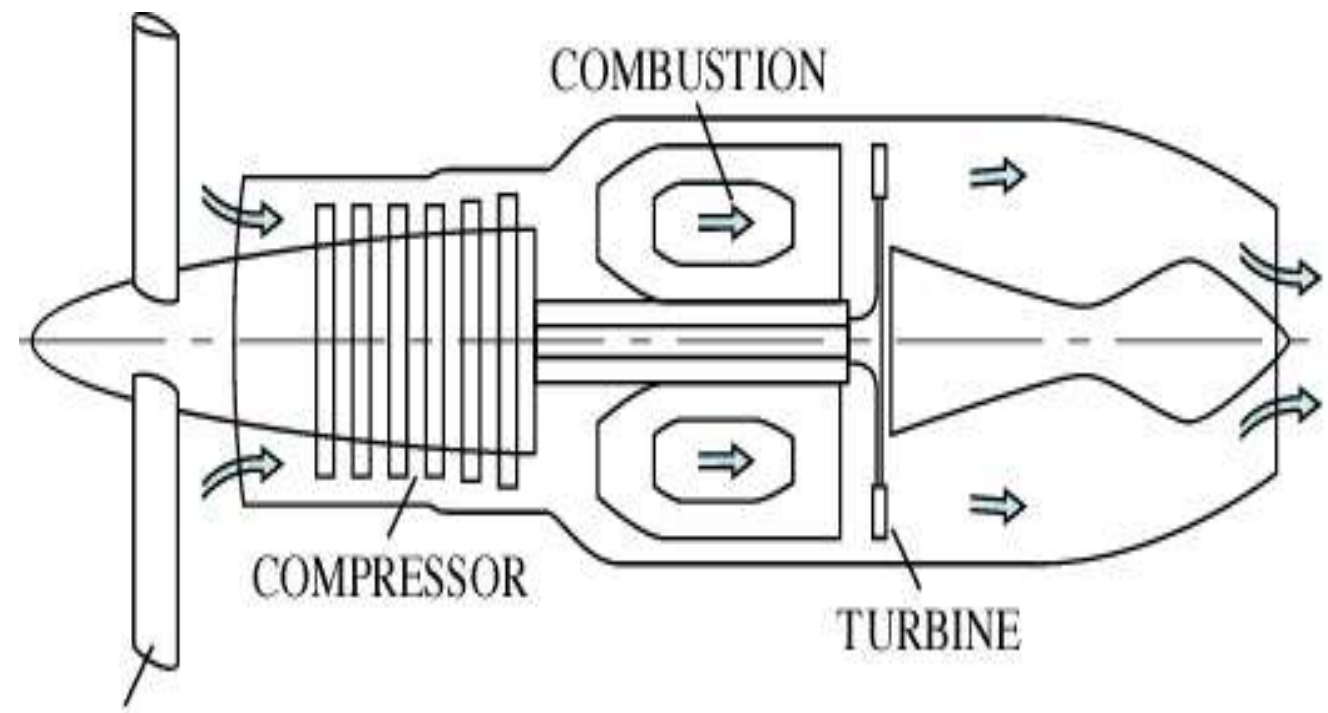

PROPELLER

Figure 7. Aircraft Turbo Propeller Engine

Let u's consider the forward path transfer function matrix and feedback path matrix of the MIMO system. 


$$
\begin{aligned}
& Y_{1}(\mathrm{~s})=G_{11}(s) R_{1}(s)+G_{12}(s) R_{2}(s) \\
& Y_{2}(\mathrm{~s})=G_{21}(s) R_{1}(s)+G_{22}(s) R_{2}(s)
\end{aligned}
$$

where $Y_{I}(s)$ is the speed of rotation of engine, $Y_{2}(s)$ is the turbine inlet temperature, $R_{I}(s)$ is the fuel rate and $R_{2}(s)$ is the propeller blade angle. Thus this engine system is a MIMO system with two inputs and outputs. In this case, $G_{11}(s)$ is the transfer function between the fuel rate and speed of rotation of engine with the propeller blade angle $R_{2}(s)$ is zero and $G_{22}(s)$ is the transfer function between the propeller blade angle and turbine inlet temperature. The high-order transfer function for this MIMO aircraft turbo propeller engine system can be written as shown in Eq. (24).

$$
G_{M}(\mathrm{~s})=\left[\begin{array}{ll}
G_{11} & G_{12} \\
G_{21} & G_{22}
\end{array}\right]=\left[\begin{array}{cc}
\frac{7}{s^{3}+1.2 s^{2}+11.8 s+7} & \frac{1}{s+2} \\
\frac{2}{3 s+2} & \frac{5}{s^{3}+5 s^{2}+7.5 s+5}
\end{array}\right]
$$

Now we can apply the presented simplification design method to the high-order aircraft MIMO system transfer function $G_{M}(s)$. In this case, we set the series term and ordering number of the fast Haar transform to 8 and time period to $0.5 \mathrm{sec}$. The approximated low order transfer function $\hat{G}_{11}(s)$ can be obtained from Eq. (25). We can apply the fast Haar transform to get the $\hat{G}_{11}(s)$.

$$
\begin{aligned}
& G_{11}(s)=\frac{1}{0.14 s^{3}+0.17 s^{2}+1.69 s+1} \\
& G_{11}(j w)=\frac{1}{0.14(j w)^{3}+0.17(j w)^{2}+1.69 j w+1}=\frac{1}{\left(1-0.17 w^{2}\right)+j w\left(1.69-0.14 w^{2}\right)} \\
& \widehat{G}_{11}(j w)=\frac{1}{1+m_{1} j w+m_{2}(j w)^{2}}=\frac{1}{\left(1-m_{2} w^{2}\right)+j w m_{1}}
\end{aligned}
$$

According to the similarity condition, $\mathrm{m} 1$ and $\mathrm{m} 2$ can be obtained by Eq. (28) and $\hat{\alpha}, \hat{\beta}, \hat{\gamma}$ and $\hat{\delta}$ are determined also.

$$
\begin{aligned}
& m_{1}=1.69-0.14 w^{2}, m_{2}=0.17 \\
& \hat{\alpha}=1, \hat{\beta}=0, \hat{\gamma}=0.17, \hat{\delta}=1.69-0.14 w^{2}
\end{aligned}
$$

We can apply the fast Haar transform to $\hat{\alpha}, \hat{\beta}, \hat{\gamma}$ and $\hat{\delta}$ for the coefficients decision process. This fast Haar transform is based on the Cooley-Tukey algorithm [12]. The fast Haar transform can also be computed using this algorithm. Figure 8 shows the CooleyTukey algorithm. 


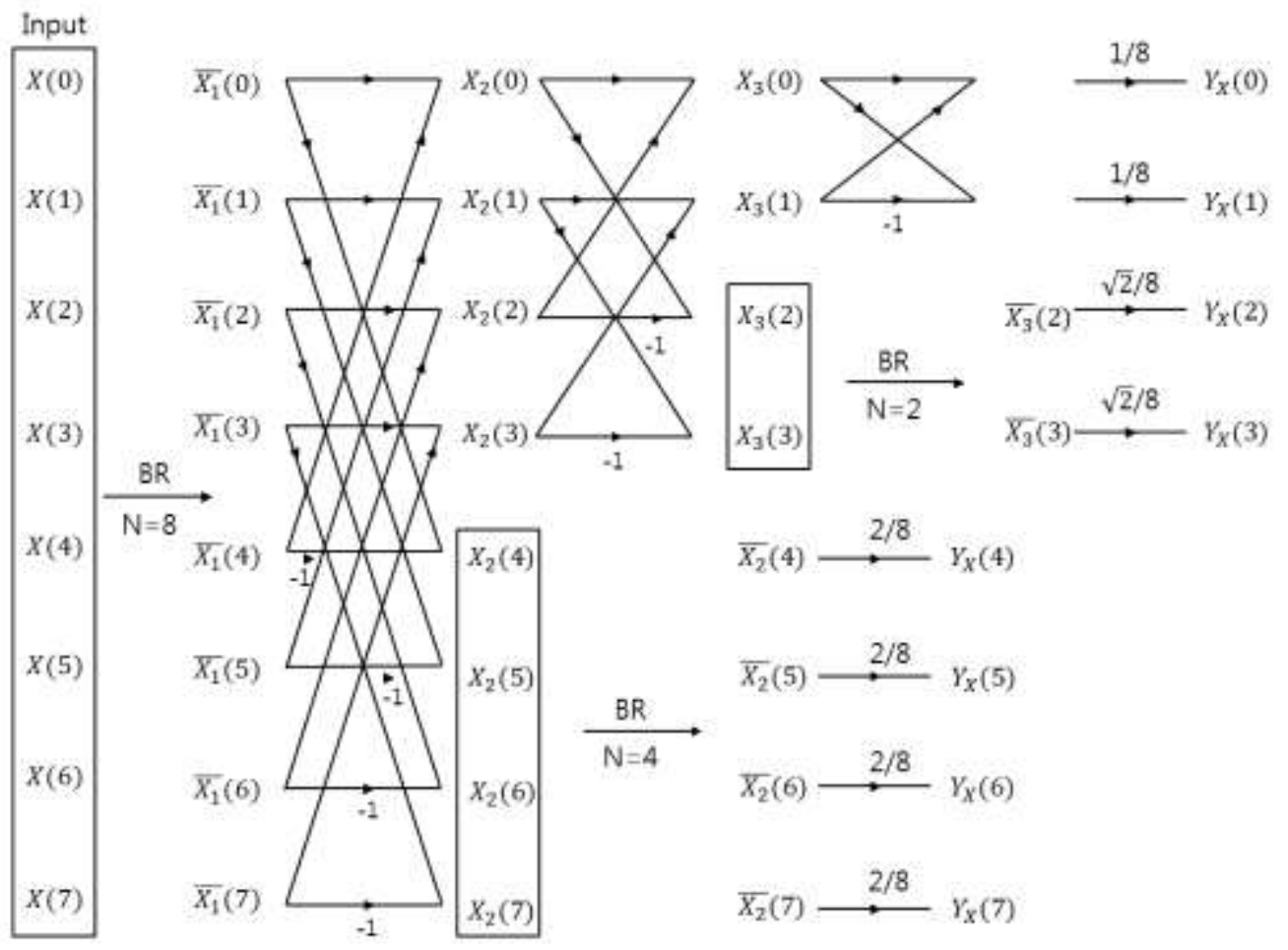

Figure 8. Computational Signal Flow Graph of the Cooley-Tukey Algorithm

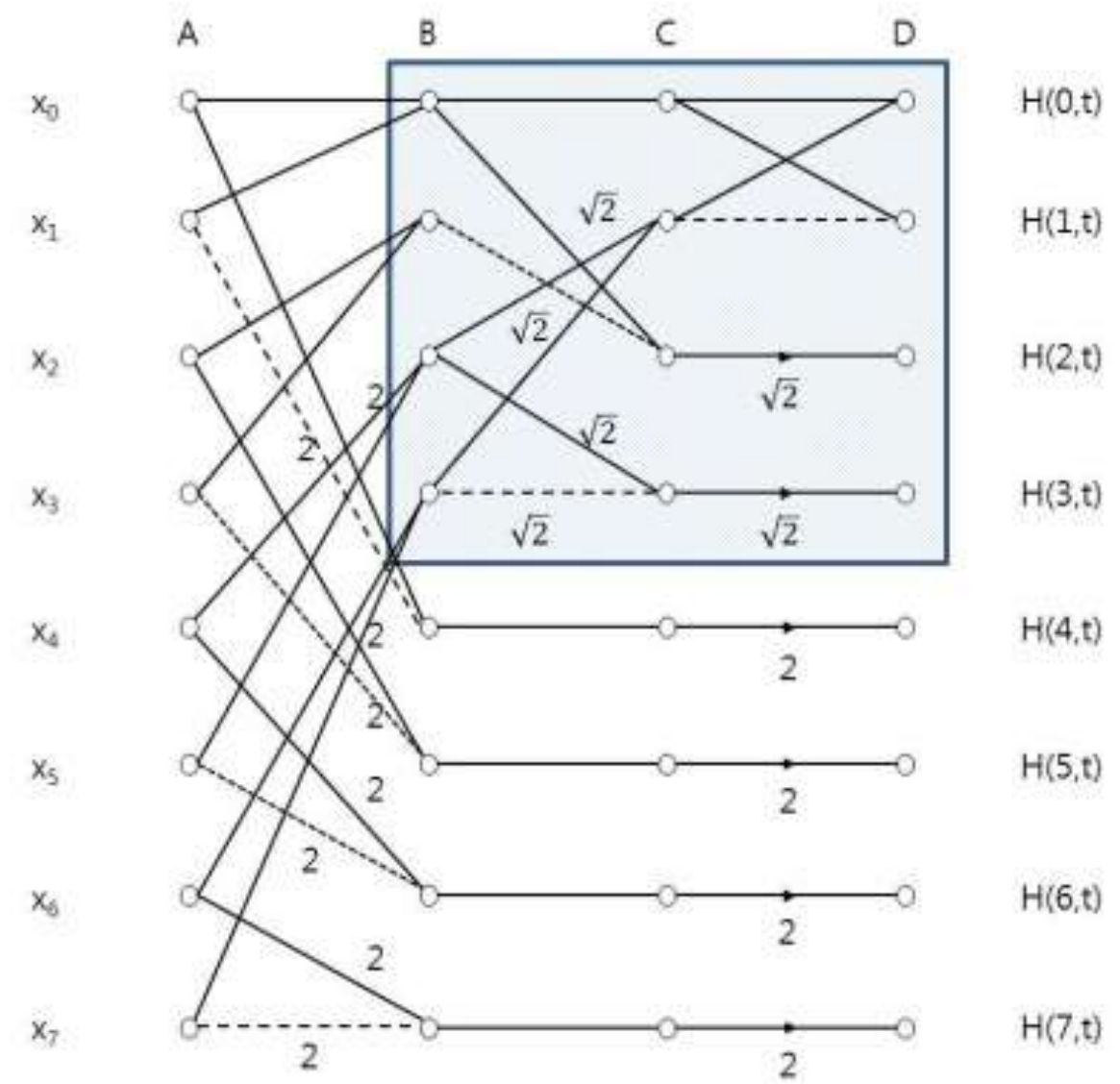

Figure 9. Fast Haar Transform for Aircraft MIMO System 
The fast Haar transform for this aircraft MIMO system is shown in Figure 9. The coefficients of the fast Haar transform are determined by addition and subtraction for three steps operations. In the first step, the addition and the subtraction value are assigned to determine the second half of four fast Haar coefficients and the first half of four fast Haar coefficients are determined through the process shown by a quadrangle. Therefore, the coefficient is determined only by the addition and subtraction process and this fast Haar transform is faster than the general Haar transform. We can get the approximated low-order transfer function $\hat{G}_{11}(\mathrm{~s})$ based on the suggested transform algorithm.

$$
\widehat{G}_{11}(\mathrm{~s})=\frac{2.1}{s^{2}+1.73 s+2.1}
$$

Now we can define the low-order transfer function $\hat{G}_{22}(s)$ from the high order transfer function of Eq. (31) using the same presented transform algorithm and process.

$$
\begin{aligned}
& G_{22}(s)=\frac{1}{0.2 s^{3}+s^{2}+1.5 s+1} \\
& \widehat{G}_{22}(j w)=\frac{1}{1.12 s^{2}+1.53 s+1}
\end{aligned}
$$

The aircraft MIMO system analysis between the original third-order system and the proposed and approximated second-order system are shown. The time domain response results are shown and the frequency domain response results are shown in Figure 10(a) and Figure 10(b) respectively. The dashed line is for the original third- order system and the dash-dotted graph displays the proposed second-order system.

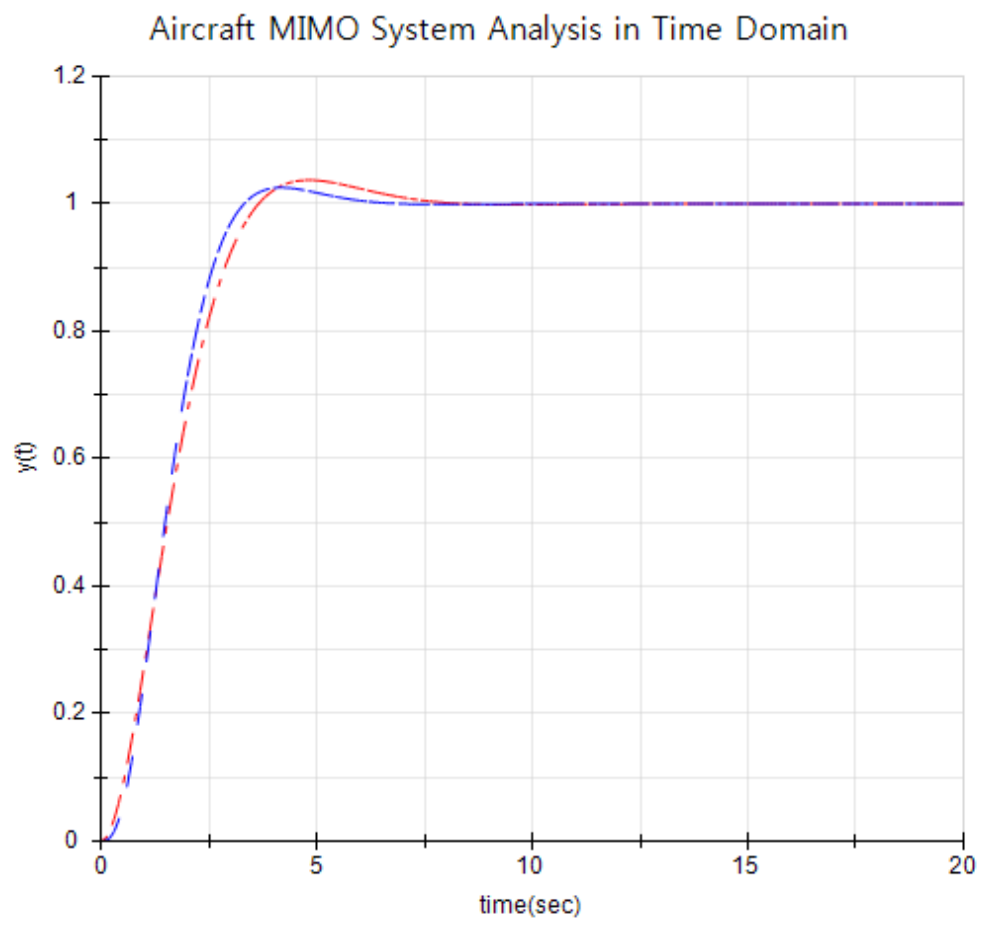

(A) Time Domain Analysis 


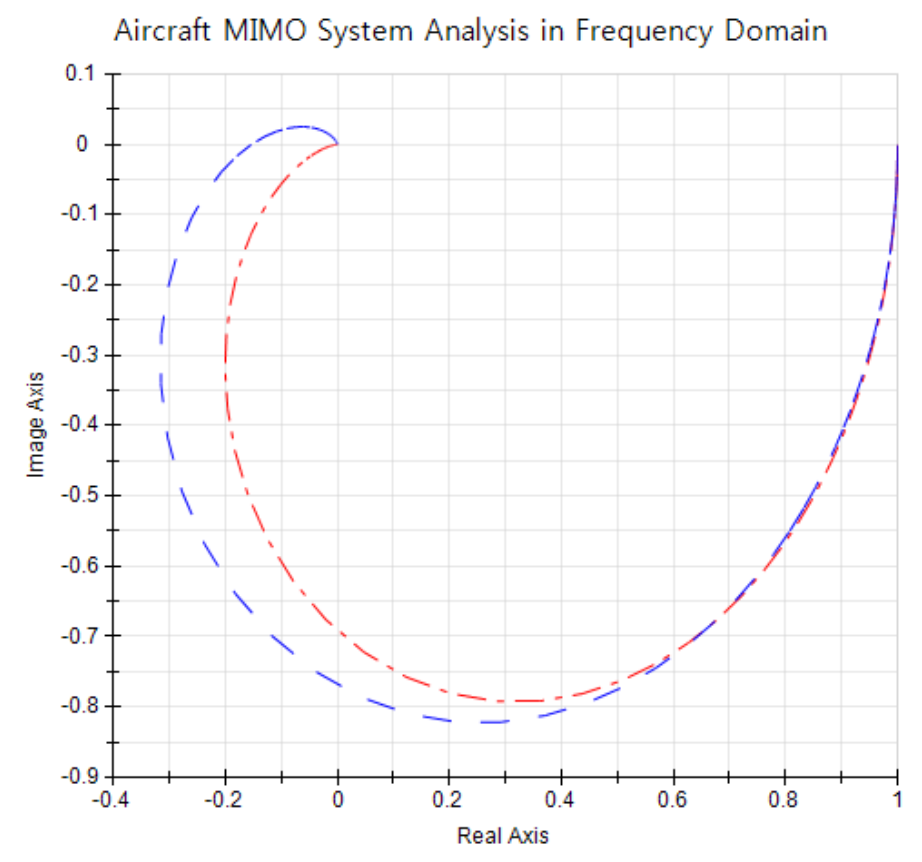

(B) Frequency Domain Analysis

\section{Figure 10. Aircraft MIMO System Analysis in Time and Frequency Domain}

\section{Conclusions}

The third-order aircraft MIMO system was approximated by a second order system using the proposed fast Haar transform algorithm. In a control system, we can obtain a reduced MIMO model by removing insignificant eigenvalues of the original MIMO system if the original MIMO system is stable. In Figure 10(a), the system time responses such as delay time, setting time and damping ratio, show almost the same results between the original third-order system and the approximated second-order system. In Figure 10(b), a slight difference in frequency responses is shown. This frequency responses gap is dependent on the initial system values and operational conditions. The fast Haar transform can reduce and save computational time by a simple addition and subtraction operation. Overall the result of the proposed method is better than the result of normal method in terms of accuracy and efficiency. Thus, the method for the high-order multivariable aircraft turbo propeller engine system presented in this paper is valuable and useful.

\section{Acknowledgement}

"This research was financially supported by the Ministry of Education (MOE) and National Research Foundation of Korea (NRF) through the Human Resource Training Project for Regional Innovation (No. 2014H1C1A1066414)."

"This was supported by Korea National University of Transportation in 2016."

\section{References}

[1] J. E. Shore, "On the applications of Haar functions", IEEE Trans. Communication, vol. 21, (1973), pp. 209-216.

[2] C.F. Chen and C. H. Hsiao, "Haar wavelet method for solving lumped and distributed parameter systems", IEE Proc. Control Theory Appl., vol. 144, no. 1, (1997), pp. 87-94. 
[3] J.H. Park and T. S. Sidhu, "Haar transform approach to distributed parameter system identification", J. of KIEE, vol.41, no.4, (2004), pp. 491-498.

[4] K. R. Rao, "A Class of discrete orthogonal transform", Int. Symp. Circuit Theory, Toronto, Canada, (1973), pp. 189-192.

[5] P. R. Roeser and M. E. Jernigan, "Fast Haar transform algorithms", IEEE Trans. Computers, vol. c-31, no. 2, (1982), pp. 175-177.

[6] J. H. Park and R.D. Oh, "Algorithm for reduction of system order using fast rationalized Haar transforms in time domain”, Int. J. of Applied Engineering Research, vo. 9, no. 23, (2014), pp. 1822318230.

[7] J. H. Park, "Application of Two Dimensional Haar Transforms and Fast Haar functions to Analysis High Order System in Time and Frequency Domain”, Int. J. of Control and Automation, vol. 7, no. 10, (2014), pp. 343-354.

[8] J. H. Park, "Simplification of high-order MIMO system using Haar transform algorithm", Advanced Science and Technology Letters, vol. 130, (2016), pp. 6-10

[9] B. C. Kuo, "Automatic Control System", (9th edition, Wiley), (2009).

[10] J.H. Park, "Transfer function approximation via rationalized Haar transform in frequency domain", Int. J. of Control and Automation, vol. 7, no. 4, (2014), pp. 247-258.

[11] J. H. Park, "Approximation algorithm for multi-input and multi-output system using generalized block pulse integration operational matrices", Int. J. of Control and Automation, vol. 8, no. 6, (2015), pp. 317326.

[12] J. W. Cooley and J. W. Tukey, "An algorithm for the machine calculation of complex Fourier series", Math. Computation, vol. 19, (1965), pp. 297-305. 